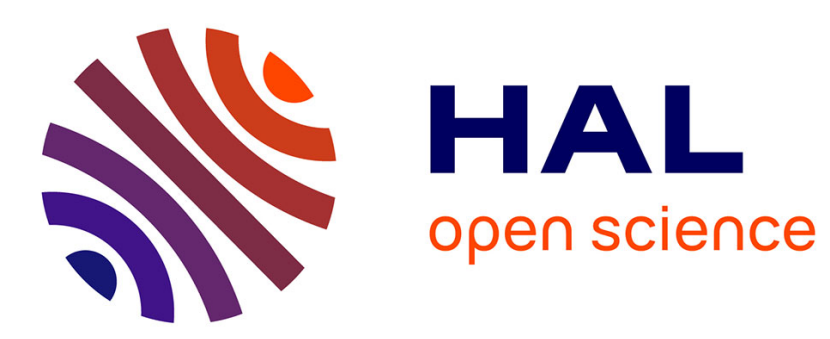

\title{
Equations of finite differences applied to torsional oscillations of crankshafts
}

\author{
Maurice A. Biot
}

\section{To cite this version:}

Maurice A. Biot. Equations of finite differences applied to torsional oscillations of crankshafts. Journal of Applied Physics, 1940, 11 (8), pp.530-537. 10.1063/1.1712808 . hal-01368630

\section{HAL Id: hal-01368630 \\ https://hal.science/hal-01368630}

Submitted on 21 Sep 2016

HAL is a multi-disciplinary open access archive for the deposit and dissemination of scientific research documents, whether they are published or not. The documents may come from teaching and research institutions in France or abroad, or from public or private research centers.
L'archive ouverte pluridisciplinaire HAL, est destinée au dépôt et à la diffusion de documents scientifiques de niveau recherche, publiés ou non, émanant des établissements d'enseignement et de recherche français ou étrangers, des laboratoires publics ou privés. 


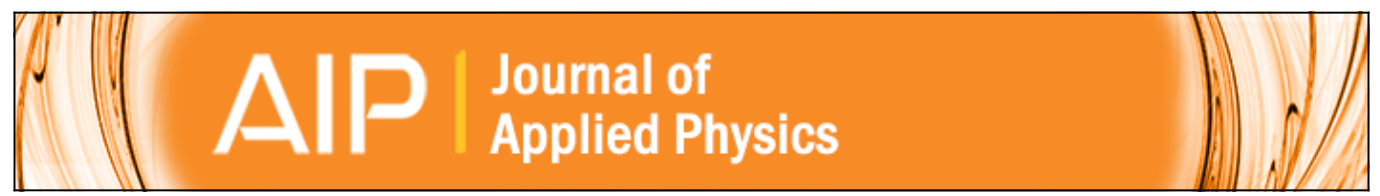

\section{Equations of Finite Differences Applied to Torsional Oscillations of Crankshafts}

Maurice A. Biot

Citation: Journal of Applied Physics 11, 530 (1940); doi: 10.1063/1.1712808

View online: http://dx.doi.org/10.1063/1.1712808

View Table of Contents: http://scitation.aip.org/content/aip/journal/jap/11/8?ver=pdfcov

Published by the AIP Publishing

\section{Articles you may be interested in}

Installation for damping torsional vibrations in a gear drive, especially between the crankshaft and an output shaft of internal combustion engines

J. Acoust. Soc. Am. 81, 2002 (1987); 10.1121/1.394737

Topological solution of ordinary and partial finite difference equations

J. Math. Phys. 21, 2475 (1980); 10.1063/1.524352

The Resistive Net and Finite-Difference Equations

Am. J. Phys. 40, 1246 (1972); 10.1119/1.1986809

On Torsional Oscillations in a Finite Circular Cylinder

J. Acoust. Soc. Am. 36, 511 (1964); 10.1121/1.1918988

Finite Difference Equations

Phys. Today 14, 64 (1961); 10.1063/1.3057516

\section{High Energy Nanosecond Lasers}
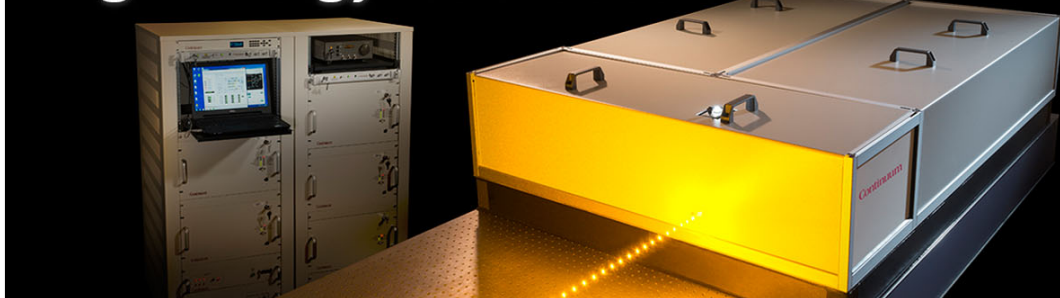
Note that this phenomenon is the same if we put

$$
S_{22}=0, \quad S_{11}=-P .
$$

The initial condition is then a horizontal tension $-P$ and the velocity of shear waves propagating in the horizontal direction is

$$
V_{h}=\left(\frac{G-\frac{1}{2} P}{\rho}\right)^{\frac{1}{2}} .
$$

If we put $G=0$ in this formula we do not find the velocity in a membrane or a string under tension. This is because the wave in a membrane is not a shear wave but a bending wave. For the viewpoint of the theory of elasticity a bending wave is the combination of two waves of the Rayleigh type at both free boundaries. ${ }^{10}$ If we calculate the velocity of these bending waves and then put $G=0$ we find

$$
V_{h}=(-P / \rho)^{\frac{1}{2}},
$$

which is the velocity of a wave in a membrane under tension $P$.

This analysis shows that propagation and reflections of elastic waves in a material under initial stress must follow laws which cannot be explained by elastic anisotropy or a change in elastic constants. In fact, because the velocity of propagation depends on the total initial shear, a discontinuity in shear may produce a reflection even if there is no discontinuity in elastic constants.

10 S. P. Timoshenko, "On the transverse vibrations of bars of uniform cross section." Phil. Mag. 43, 125 (1922).

\title{
Equations of Finite Differences Applied to Torsional Oscillations of Crankshafts*
}

\author{
Maurice A. Biot \\ Columbia University, New York, New York
}

(Received April 8, 1940)

From the viewpoint of torsional oscillations an internal combustion engine with a long crankshaft is generally considered to be equivalent to a uniform shaft carrying equidistant identical disks. It is here shown that advantage can be taken of the regularity of such a system to simplify the calculation of torsional oscillations. This is done by applying a mathematical method known as the calculus of finite differences. The procedure leads to a frequency equation (2.7) of remarkable symmetry in which appear as parameter the number $n$ of cylinders in line and two simple functions $K_{1}$ and $K_{2}$ of the frequency which characterize the dynamical properties of the machines coupled at both ends of the crankshaft. These characteristic functions are

\section{Mechanical Impedance and Dynamic Modulus}

$\coprod \mathrm{N}$ $\checkmark$ the theory of electric networks the concept of impedance has proved to be a highly useful tool for both the analytical treatment and the comprehension of electrical phenomena. Its use has been extended to the field of mechanics by the introduction of so-called equivalent networks or, as in acoustics, by defining the mechanical impedance as the ratio of force to velocity. The

\footnotetext{
* Publication assisted by the Ernest Kempton Adams Fund for Physical Research of Columbia University.
}

of the nature of mechanical impedances, but due to their physical interpretation as a spring modulus. (or spring constant) generalized to dynamic phenomena, the appellation dynamic modulus is being preferably used in the present paper. The concept of dynamic modulus is briefly introduced in the first section, while the second deals with the establishment of the frequency equation and an artifice for its rapid graphical solution avoiding the necessity of plotting an oscillatory function. Numerical applications to Diesel engines are treated in the last section. An example is also given of an extreme case where the fundamental frequency has a very low value and a special method is used for the calculation of this frequency.

latter definition is very useful in compound electromechanical systems and in those for which the amount of dissipated or radiated energy is one of the important features. However, in systems without or with negligible dissipation constituted, for instance, by a combination of masses and springs, it seems preferable to introduce as mechanical impedance the ratio of force to displacement. When there is no dissipation this ratio is a real quantity which can be either positive or negative. It generalizes the concept of spring constant to the case of harmonic mo- 
tion. In order to distinguish this type of impedance from the other we use the appellation dynamic spring constant or simply dynamic modulus. The following example illustrates the above definition.*

An elastic rod $A B$ of torsional spring constant $k$ is clamped at $B$ and carries at $A$ a disk of moment of inertia $I$ (Fig. 1). In order to produce an harmonic oscillation of the disk of frequency $\omega / 2 \pi$ and of amplitude $\theta$ we must apply to the disk a torque

$$
M=\left(k-I \omega^{2}\right) \theta .
$$

This may be written

with

$$
M=K \theta
$$

$$
K=k+I p^{2} \quad p=i \omega \quad i=\sqrt{ }-1 .
$$

The quantity $K$ is the dynamic modulus of the system at $A$. It becomes equal to the static modulus $k$ of the rod for small frequencies, it vanishes at resonance and is negative for higher frequencies.

In the case of a shaft carrying two disks and free to rotate in bearings as illustrated in Fig. 2, it is readily verified that the dynamic modulus $K_{2}$ at the left end is obtained by the following steps. The dynamic modulus $K^{\prime}$ at the left of

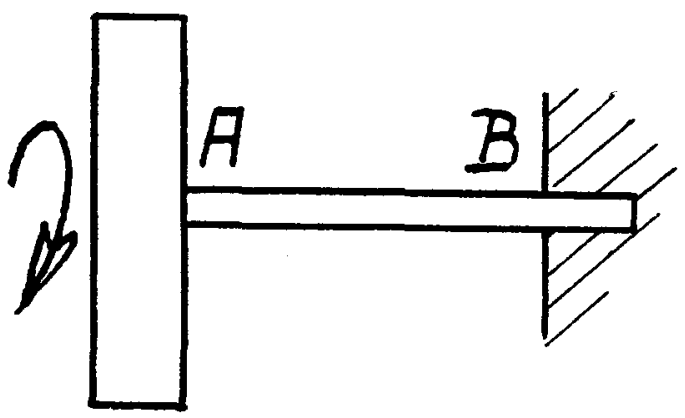

Frg. 1. Example of mechanical impedance.

the system $(k, I)$ alone is given by

$$
1 / K^{\prime}=1 / k+1 / I p^{2} \text {. }
$$

If we add the mass $I_{1}$ the dynamic modulus becomes $K^{\prime \prime}$

$$
K^{\prime \prime}=K^{\prime}+I_{1} p^{2}
$$

* Further developments on mechanical impedances, the concept of dynamic modulus and the application of the calculus of finite differences to engineering problems will be found in a textbook by Dr. Th. von Karman and the author (Mathematical Methods in Engineering (McGraw Hill Book Co., Inc., New York, 1940)). and adding the spring $k_{1}$ the dynamic modulus $K_{2}$ is given by

$$
1 / K_{2}=1 / k_{1}+1 / K^{\prime \prime} .
$$

The law of formation of the dynamic modulus $\dagger$ is the well-known series-parallel combination rule for the electrical impedance of a ladder-type

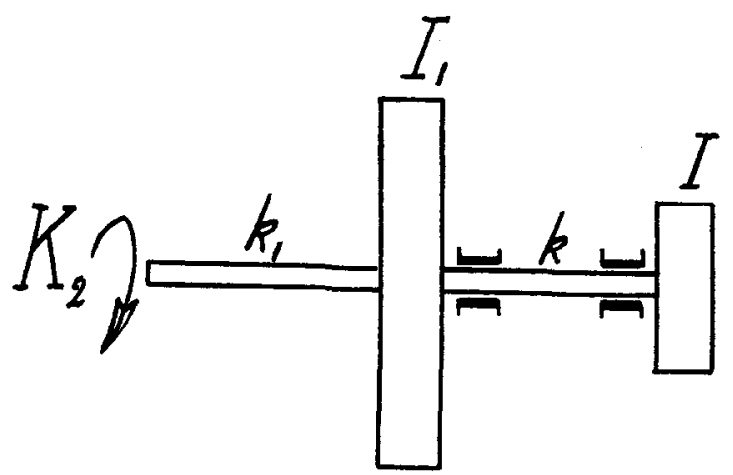

Frg. 2. Example of mechanical impedance.

network. From (1.2), (1.3), (1.4) we find

$$
K_{2}=\frac{1}{\left[\frac{1}{k_{1}}+\frac{1}{-I_{1} \omega^{2}+\frac{1}{1 / k-1 / I \omega^{2}}}\right]}
$$

The physical meaning of this quantity $K_{2}$ is that a harmonic torque of given frequency $\omega / 2 \pi$ acting on the left end of the system produces an amplitude at that point which would be the same as if the torque were driving an elastic rod clamped at the other end and of spring constant equal to $K_{2}$. Also if we put $K_{2}=0$ we obtain the frequency equation for the free oscillations of the system, while $1 / K_{2}=0$ is the frequency equation when it is rigidly clamped at the left end.

\section{Frequency Equation}

Using the concept of dynamic modulus and a mathematical method known as the calculus of finite differences we are now going to show that it is possible to establish a frequency equation of remarkable simplicity.

Following the usual procedure we represent

t A similar rule for the combination of springs is indicated in J. P. Den Hartog, Mechanical Vibrations (McGraw Hill Book Co., New York, 1934), p. 41. 

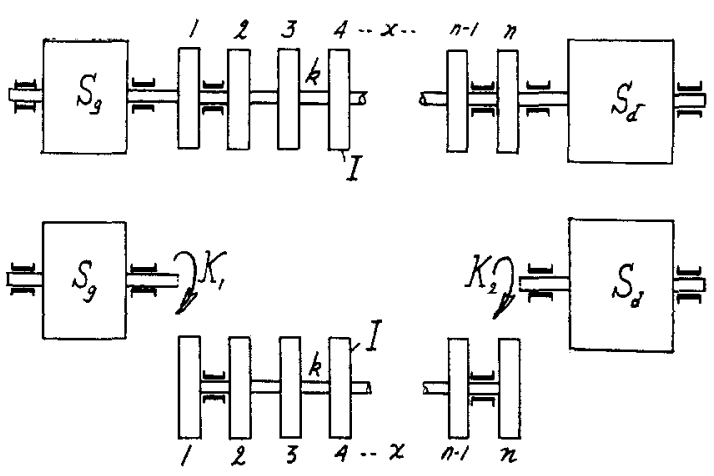

FIG. 3. Schematic representation of an internal combustion engine and its end impedances.

schematically the crankshaft rods and pistons by a uniform shaft carrying as many disks as there are cylinders in line. (Fig. 3). Let $n$ be the number of disks (or cylinders in line) numbered from 1 to $n, I$ their moment of inertia and $\theta_{x}$ the amplitude of oscillation of a disk numbered $x$. It is well known that the amplitudes of three successive disks satisfy the equation

$$
I \omega^{2} \theta_{x}=k\left(-\theta_{x-1}+2 \theta_{x}-\theta_{x+1}\right),
$$

where $\omega$ is the angular frequency of the oscillation and $k$ the torsional spring constant of a section of the shaft between two disks.* If we put $\alpha^{2}=I \omega^{2} / k$ Eq. (2.1) may be written

$$
\theta_{x-1}-\left(2-\alpha^{2}\right) \theta_{x}+\theta_{x+1}=0 .
$$

There are $n-2$ such equations for $x=2,3, \cdots$, $n-1$. In order to express the corresponding relations for the end disks we assume that a machine of dynamic modulus $K_{1}$ is coupled at the left end of the shaft and a machine of dynamic modulus $K_{2}$ at the right end. Then the amplitudes of oscillation $\theta_{1}$ and $\theta_{2}$ satisfy the equations,

$$
\begin{aligned}
\theta_{2}-\left(1-\alpha^{2}+K_{1} / k\right) \theta_{1} & =0 \\
\theta_{n-1}-\left(1-\alpha^{2}+K_{2} / k\right) \theta_{n} & =0 .
\end{aligned}
$$

The recurrence Eq. (2.2) is called an equation with finite differences of the second order. Such an

\footnotetext{
* As pointed out by R. Grammel [see for instance: über einige dynamische Probleme bei Kolben motoren Schriften der Deutschen Akademie der Luftfohrtforschung (1939)] due to the existence of play between bearings and crankshaft journals, the model should include a slight coupling between the disks $x$ and $x+2$. This can also be treated by the method of finite differences. It is believed however that the classical method of perturbations might yield more quickly the corrected modes and frequencies. Another correction which can be obtained by the method of perturbations is due to the fact that the moments of inertia of the disks include a periodic function of time.
}

equation may be treated by methods similar to those used in the solution of differential equations, with relations (2.3) playing here the role of "boundary conditions."

Let us first assume that $|\alpha|<2$. In that case the so-called general solution of Eq. (2.2) is known to be

$$
\theta_{x}=A \cos \mu x+B \sin \mu x,
$$

where $A$ and $B$ are arbitrary constants and $\mu$ is related to the frequency by the relation

$$
\omega=2(k / I)^{\frac{1}{3}} \sin \mu / 2 .
$$

The arbitrary constants $A$ and $B$ are determined by substituting the general solution (2.4) into the boundary conditions (2.3). Putting $K_{1} / k-1=M$, $K_{2} / k-1=N$ we find

$$
\begin{gathered}
A(1+M \cos \mu)+B M \sin \mu=0 \\
A[\cos \mu(n+1)+N \cos \mu n] \\
+B[\sin \mu(n+1)+N \sin \mu n]=0 .
\end{gathered}
$$

These are two simultaneous equations for $A$ and $B$. A more detailed derivation is given in the Appendix at the end of the paper. The elimination of $A$ and $B$ between these two equations yields the frequency equation,

$$
\begin{aligned}
& \sin \mu(n+1)+(M+N) \sin \mu n \\
& +M N \sin \mu(n-1)=0 .
\end{aligned}
$$

We have assumed $|\alpha|<2$ or $\omega=2(k / I)^{\frac{1}{2}}$ which means that the frequency Eq. (2.7) yields only the natural frequencies of the system which are lower than $2(k / I)^{\frac{1}{2}}$. In most practical cases however this range will be sufficient as it will actually cover all the frequencies of the system or at least the greatest number of them and the most important ones. The limiting value $\omega_{c}=2(k / I)^{\frac{1}{2}}$ of the frequency has an important physical significance as regards the response of the crankshaft to vibrations. In the terminology of electric wave filters $\omega_{c}$ is called the cut-off frequency. In fact the crankshaft is the mechanical equivalent of a low pass filter and it reflects all torsional waves of frequency higher than the cut-off frequency. For a frequency approaching this limiting value the mode of vibration in the crankshaft is such that two successive cranks oscillate in opposite phases. 
In principle, in order to find the roots of the frequency equation it would be sufficient to plot the left side of Eq. (2.7) as a function of $\mu$ or $\omega$ and note the abscissas where the curve intersects the horizontal axis. This procedure however, is generally cumbersome because the function to plot is highly oscillatory and requires the calculation of a great number of points. This difficulty may be avoided and the numerical work considerably simplified by the following artifice.

We notice that the frequency equation may be written in complex form by putting

$$
\begin{aligned}
e^{\mu i / 2}+M e^{-\mu i / 2} & =A_{1} e^{\varphi_{1} i} \\
e^{\mu i / 2}+N e^{-\mu i / 2} & =A_{2} e^{\varphi_{2} i} .
\end{aligned}
$$

The left side of the frequency Eq. (2.7) is then the imaginary part of $A_{1} A_{2} e^{\left(\mu n+\varphi_{1}+\varphi_{2}\right) i}$. An equivalent form of the frequency equation is therefore

$$
\mu n+\varphi_{1}+\varphi_{2}=\text { multiple of } \pi,
$$

where $\varphi_{1}$ and $\varphi_{2}$ are functions of $\mu$ given by

$$
\begin{aligned}
& \varphi_{1}=\tan ^{-1}\left[\left(\frac{2 k}{K_{1}}-1\right) \tan \frac{\mu}{2}\right], \\
& \varphi_{2}=\tan ^{-1}\left[\left(\frac{2 k}{K_{2}}-1\right) \tan \frac{\mu}{2}\right] .
\end{aligned}
$$

In Eq. (2.9) the left side represents a smooth function of $\mu$ generally near to a straight line. This form of the frequency equation is therefore well fit for solution by graphical methods and interpolation. The procedure will be made clear by the numerical examples below.

The method is of course not limited to the calculation of frequencies lower than the cut-off frequencies. If $|\alpha|>2$ the general solution of the difference equation (2.2) is

$$
\theta_{x}=A(-1)^{x} \cosh \mu x+B(-1)^{x} \sinh \mu x,
$$

where $\mu$ is related to $\omega$ by the relation,

$$
\omega=2(k / I)^{\frac{1}{2}} \cosh \mu / 2 .
$$

Proceeding as we have done above this leads to a frequency equation similar to (2.6) this time with hyperbolic instead of circular function.

The shape of the modes of vibration in the crankshaft may be calculated below the cut-off frequency by (2.4) and above by (2.11). Using, for instance, the first condition (2.6) we may write (2.4) in the form

with

$$
\theta_{x}=C \sin (\mu x+\beta)
$$

$$
\tan \beta=\left(1-K_{1} / k\right) \sin \mu /\left[1+\left(K_{1} / k-1\right) \cos \mu\right]
$$

and $C$ an arbitrary constant. The torsional mode of order $r$ is found by substituting the values $\mu_{r}$ and $\omega_{r}$, roots of the frequency equation and corresponding to that mode.

\section{Example 1}

\section{Applications}

We consider the case of a crankshaft with $n$ cranks free at both ends. Then $K_{1}=K_{2}=0$. The frequency Eq. (2.7) may be written

$$
\begin{aligned}
\sin \mu(n+1)-2 \sin \mu n+\sin \mu(n+1) & =0 \\
\text { or } \quad \sin \mu n & =0 .
\end{aligned}
$$

The roots are $\mu=p \pi / n(p=0,1,2, \cdots, n)$. The values $p=0$ and $p=n$ must be excluded because they do not correspond to any motion of the crankshaft. The $(n-1)$ natural frequencies are therefore given by the formula

$$
\omega_{p}=\omega_{c} \sin p \pi / n, \quad p=1,2, \cdots, n-1 .
$$

The spectrum of frequencies is represented in Fig. 4. We notice that the highest frequencies have a tendency to gather in the vicinity of the

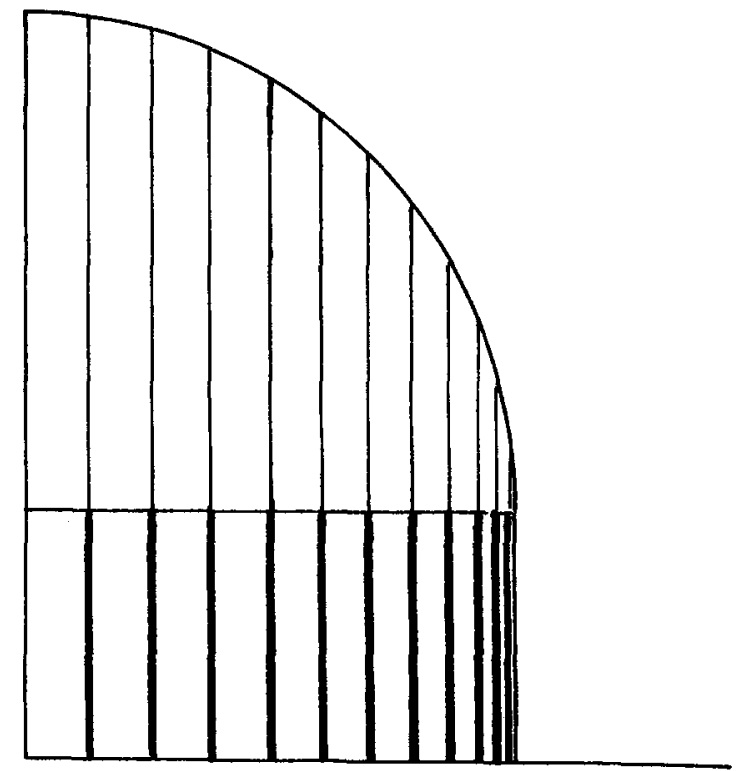

FIG. 4. Frequency spectrum of a 12-cylinder in-line engine without end impedances. 


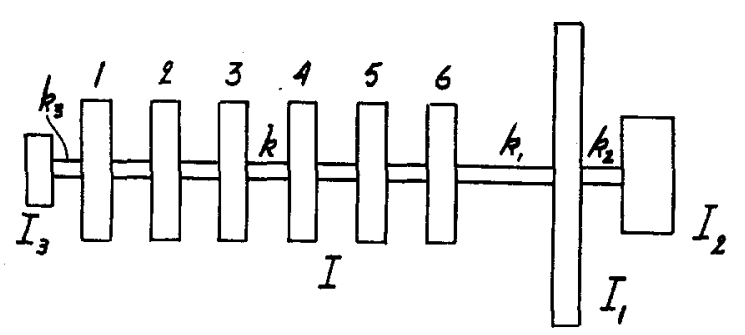

FIG. 5. Six-cylinder Diesel with pump flywheel and pulley.

cut-off frequency $\omega_{c}=2(k / I)^{\frac{1}{2}}$. This is a general feature in the oscillations of long crankshafts. It is also of interest to consider the frequency equation in the form (2.9). We may write in this case $\varphi_{1}=\varphi_{2}=-\pi / 2$ then the Eq. (2.9) becomes

$$
\mu n=\text { multiple of } \pi \text {. }
$$

In this case the left side of the equation as a function of $\mu$ represents a straight line.

\section{Example 2}

A six-cylinder Diesel installation is represented in Fig. 5. The numerical data are:*

$I=3920$ lb. in. sec. ${ }^{2}$

$I_{1}=139800 \mathrm{lb}$. in. sec. ${ }^{2}$

$I_{2}=26400 \mathrm{lb}$. in. sec. ${ }^{2}$

$I_{3}=708 \mathrm{lb}$. in. sec. ${ }^{2}$

The dynamic modulus $K_{1}$ at the left end of the crankshaft is given by

$$
1 / K_{1}=1 / k_{3}-1 / I_{3} \omega^{2}
$$

and $2 k / K_{1}$ expressed as a function of $\mu$ by means of relation (2.5) is

$$
\frac{2 k}{K_{1}}=\frac{2 k}{k_{3}}-\frac{I}{2 I_{3}} \frac{1}{\sin ^{2} \mu / 2} .
$$

Introducing this in the first formula (2.10) we calculate $\varphi_{1}$ as a function of $\mu$. In the same way we calculate $2 k / K_{2}$ where $K_{2}$ is the dynamic modulus at the right end of the crankshaft. This may be calculated as a function of $\mu$ in the following steps

$$
\begin{aligned}
\omega^{2} & =4 k / I \sin ^{2} \mu / 2 \\
1 / K^{\prime} & =1 / k_{2}-1 / I_{2} \omega^{2} \\
K^{\prime \prime} & =-I_{1} \omega^{2}+K^{\prime} \\
2 k / K_{2} & =2 k / k_{1}+2 k / K^{\prime \prime} .
\end{aligned}
$$

* The numerical data for this engine are taken from $\mathrm{S}$. Timoshenko, Vibration Problems in Engineering (D. Van Nostrand, New York, 1939), p. 150.
Introducing this function in the second formula (2.10) we calculate $\varphi_{2}$. Calculations need only be made in the range $0<\mu<\pi$. The frequency equation

$$
6 \mu+\varphi_{1}+\varphi_{2}=\text { multiple of } \pi
$$

will be solved graphically by plotting the left side as a function of $\mu$. The values of the functions $\varphi_{1}, \varphi_{2}$ are given in Table I.

The curve representing $6 \mu+\varphi_{1}+\varphi_{2}$ is plotted in Fig. 6; the intersections of this curve with the horizontals of ordinates $0, \pi, 2 \pi, 3 \pi, 4 \pi, 5 \pi$, yield the roots of the frequency equation (3.6). These roots are in degrees,

$$
\begin{array}{lll}
\mu_{1}=12.75 & \mu_{2}=37.5 & \mu_{3}=63.7 \\
\mu_{4}=91.2 & \mu_{5}=129 & \mu_{6}=149 .
\end{array}
$$

The corresponding frequencies are derived from the formula $\omega=\omega_{c} \sin \mu / 2$ where $\omega_{c}=2(k / I)^{\frac{3}{2}}=866$; the natural frequencies are

$$
\begin{aligned}
& f_{1}=\frac{866}{2 \pi} \sin \frac{12.75}{2}=15.3 \mathrm{sec} .^{-1} \\
& f_{2}=\frac{866}{2 \pi} \sin \frac{37.5}{2}=44.3 \mathrm{sec} .^{-1} \\
& f_{3}=\frac{866}{2 \pi} \sin \frac{63.7}{2}=72.4 \mathrm{sec} .^{-1} \\
& f_{4}=\frac{866}{2 \pi} \sin \frac{91.2}{2}=98.3 \mathrm{sec} .^{-1} \\
& f_{5}=\frac{866}{2 \pi} \sin \frac{129}{2}=124 \mathrm{sec} .^{-1} \\
& f_{6}=\frac{866}{2 \pi} \sin \frac{149}{2}=132 \mathrm{sec} .^{-1} .
\end{aligned}
$$

TABLE I.

\begin{tabular}{r|c|c|c}
\hline \multicolumn{1}{c|}{} & $\varphi 1$ & $\varphi_{2}$ & $6 \mu+\varphi_{1}+\varphi_{2}$ \\
\hline 0 & -90 & -90 & -180 \\
5 & -8.20 & -89.3 & -67.5 \\
10 & 5.75 & -88.2 & -22.4 \\
15 & 14.7 & -87.3 & 17.4 \\
20 & 22.1 & -86.3 & 55.7 \\
30 & 34.8 & -83.7 & 131 \\
45 & 46.5 & -82.2 & 234 \\
60 & 56.0 & -79.5 & 336 \\
75 & 63.5 & -76.3 & 437 \\
90 & 69.1 & -75.4 & 533 \\
105 & 73.6 & -74.1 & 629 \\
120 & 77.6 & -73.6 & 724 \\
150 & 84.1 & -78.2 & 905 \\
280 & 90 & -90 & 1080 \\
\hline
\end{tabular}


These are the six natural frequencies of the system below the cut-off frequency whose value is in this case $138 \mathrm{sec}^{-1}$. The system has actually eight natural frequencies, there are therefore two more frequencies higher than 138 but their practical importance is not great. They could however eventually be calculated by the procedure indicated above [cf. Eq. (2.11)].

The complete calculation of the six frequencies requires about two or three hours of slide rule work. The method is very well fit to discuss rapidly the effect on the frequencies of a structural change of the system. If, for example, the

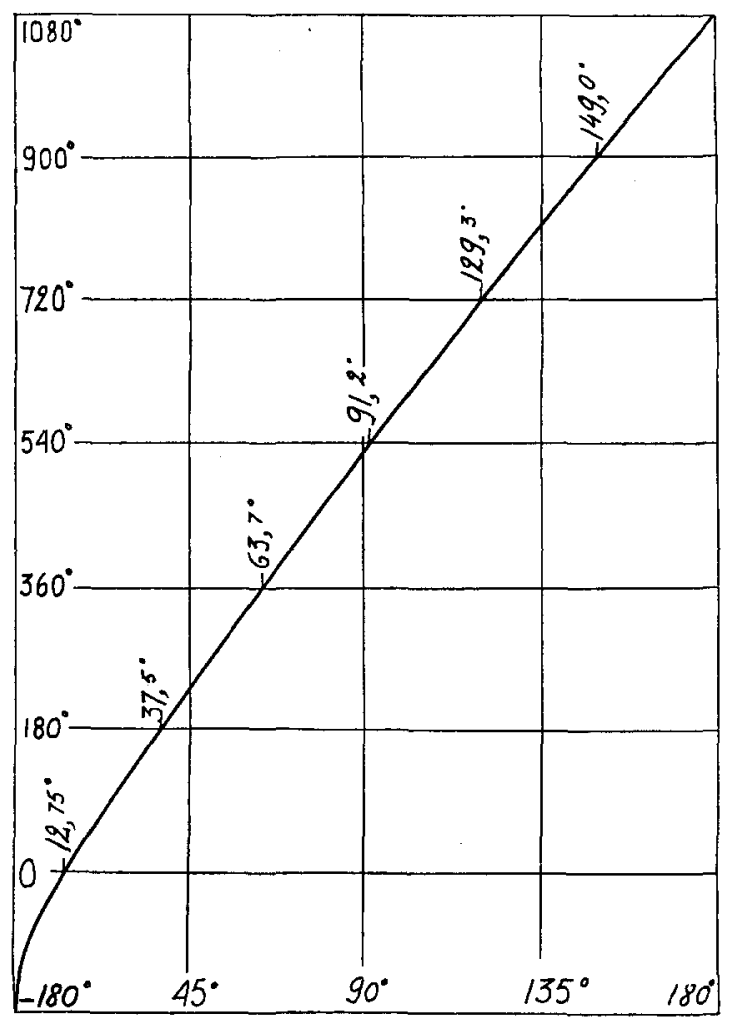

FIG. 6. The function $6 \mu+\varphi_{1}+\varphi_{2}$ for the engine in Fig. 5 plotted as function of $\mu$.

number of cylinders were 12 instead of 6 , the 12 natural frequencies below 138 would be obtained without repeating all the calculations by solving the equation

$$
12 \mu+\varphi_{1}+\varphi_{2}=\text { multiple of } \pi,
$$

where the functions $\varphi_{1}$ and $\varphi_{2}$ are numerically the same as above.

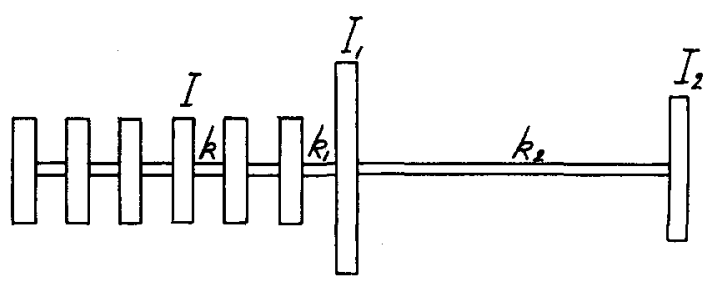

FIG. 7. A six-cylinder Diesel ship drive with flywheel and propeller.

\section{Example 3}

We consider the six-cylinder Diesel ship drive represented in Fig. 7. The propeller is driven by a long shaft. The data are,*

$$
\begin{aligned}
k & =k_{1}=675 \times 10^{6} \mathrm{in} . \mathrm{lb} . / \mathrm{rad} . \\
k_{2} & =13.5 \times 10^{6} \text { in. } \mathrm{lb} . / \mathrm{rad} . \\
I & =2560 \mathrm{lb} . \text { in. sec. } \\
I_{1} & =75000 \mathrm{lb} . \text { in. sec. } .^{2}
\end{aligned}
$$

Since $K_{1}=0$ we may take for $\varphi_{1}$ the constant value $\varphi_{1}=-90^{\circ}$ while $\varphi_{2}$ is calculated as a function of $\mu$ as in the previous example. We find the values given in Table II.

The curve representing $6 \mu-90+\varphi_{2}$ as a function of $\mu$ is plotted in Fig. 8. The intersections of this curve with the horizontals of ordinates 0 , $\pi, 2 \pi, 3 \pi, 4 \pi, 5 \pi, 6 \pi$ yield seven roots of the frequency $\mathrm{Eq} .(2.8)$

$$
\begin{array}{lll}
\mu_{2}=14.9 & \mu_{3}=42.0 & \mu_{4}=69.5 \\
\mu_{5}=97.0 & \mu_{6}=124.5 & \mu_{7}=152 .
\end{array}
$$

The lowest root $\mu_{1}$ determined by the horizontal of abscissa 0 cannot be evaluated easily by this method unless the function $\varphi_{2}$ is calculated very accurately in the interval $0-5^{\circ}$. This is not very convenient and it is preferable in this case to use directly the frequency equation in the form (2.7). Since $K_{1}=0$ it may be written

$$
\omega^{2} I \frac{\sin \mu n}{2 \sin (\mu / 2) \cos \mu\left(n-\frac{1}{2}\right)}=K_{2} .
$$

For small values of $\mu$ the factor

$$
\lambda(\mu)=\frac{\sin \mu n}{2 \sin (\mu / 2) \cos \mu\left(n-\frac{1}{2}\right)}
$$

is approximately equal to $n$ and the frequency equation becomes

$$
n \omega^{2} I=K_{2}
$$

* The numerical data of this engine are taken from J. P. Den Hartog, Mechanical Vibrations (McGraw Hill Book Co., New York, 1934), p. 208. 


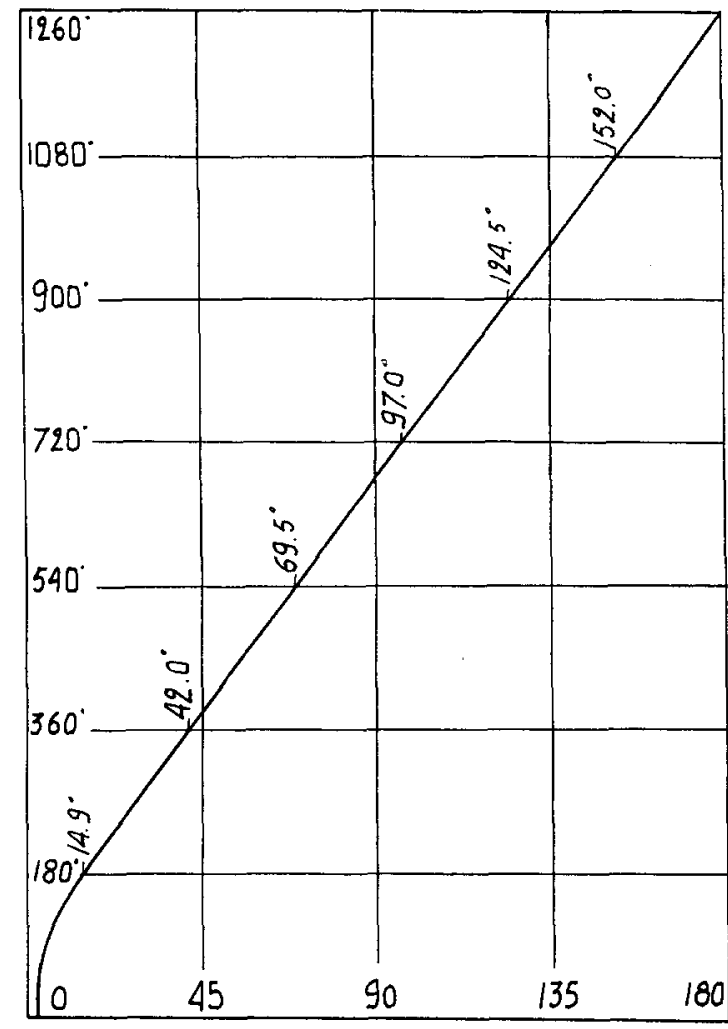

FIG. 8. The function $6 \mu+\varphi_{1}+\varphi_{2}$ for the engine in Fig. 7 plotted as function of $\mu$.

It is clear that this equation is the frequency equation for the case of a rigid crankshaft. The factor $\lambda$ plays the role of a correction factor taking into account the elasticity of the crankshaft. Introducing the numerical data and putting $z=2 \sin \mu / 2$ the frequency equation may be written

$$
0.02 \lambda+0.7736-(10.15 \lambda+275) z^{2}+275 \lambda z^{4}=0 .
$$

A first approximation for the lowest root is found by putting $\lambda=6$. Neglecting the term in $z^{4}$ we find

$$
\begin{aligned}
& z^{2}=\frac{0.02 \times 6+0.7736}{10.15 \times 6+275}=2.65 \times 10^{-3} \\
& z=5.15 \times 10^{-2}
\end{aligned}
$$

This corresponds to an angle $\mu=2.95^{\circ}$. Introducing this value of $\mu$ in the factor $\lambda$ we find $\lambda=6.09$. This yields the second approximation

$$
\begin{aligned}
z^{2} & =\frac{0.02 \times 6.09+0.7736+6.09 \times 275 \times(2.65)^{2} 10^{-6}}{10.15 \times 6.09+275}, \\
z & =5.19 \times 10^{-2}, \quad \mu_{1}=2.98^{\circ} .
\end{aligned}
$$
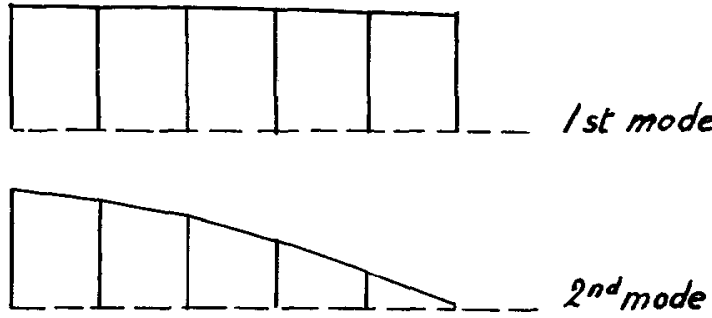

$2^{n d}$ mode

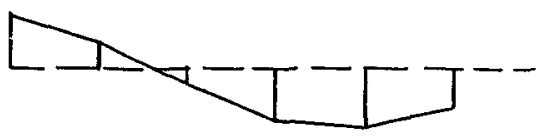

$3^{d}$ mode

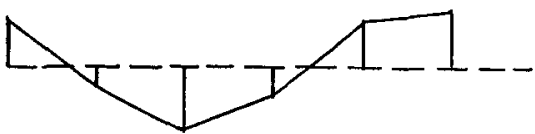

$4^{\text {th }}$ mode

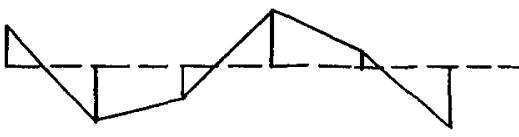

5thmode

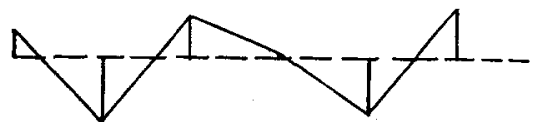

6"mode

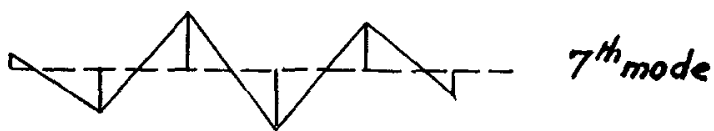

FIG. 9. The shape of the torsional modes in the crankshaft of the engine in Fig. 7 for the seven natural frequencies of the system.

This second approximation is quite satisfactory.

The lowest frequency is

$$
f_{1}=\frac{z}{2 \pi}\left(\frac{k}{I}\right)^{\frac{1}{2}}=\frac{5.19 \times 10^{-2}}{2 \pi} \times 512=4.24 \mathrm{sec}^{-1} \text {. }
$$

TABLE II.

\begin{tabular}{rcc}
\hline$\mu$ & $\varphi 2$ & $6 \mu-90+\varphi_{2}$ \\
\hline 0 & -90 & -180 \\
5 & 158.1 & 98.1 \\
10 & 173.8 & 143.8 \\
15 & 180.07 & 180.07 \\
20 & 184.35 & 214.35 \\
30 & 191.65 & 281.65 \\
45 & 200.0 & 380.0 \\
60 & 208.2 & 478.2 \\
75 & 216.1 & 576.1 \\
90 & 224.1 & 674.1 \\
105 & 231.6 & 771.6 \\
120 & 239.4 & 869.4 \\
150 & 254.7 & 1064.7 \\
180 & 270 & 1260 \\
\hline
\end{tabular}


The other frequencies are

$$
\begin{aligned}
& f_{2}=\frac{1024}{2 \pi} \sin \frac{14.9}{2}=21.2 \mathrm{sec} .^{-1} \\
& f_{3}=\frac{1024}{2 \pi} \sin \frac{42.0}{2}=58.4 \mathrm{sec}^{-1} \\
& f_{4}=\frac{1024}{2 \pi} \sin \frac{69.5}{2}=92.8 \mathrm{sec}^{-1} \\
& f_{5}=\frac{1024}{2 \pi} \sin \frac{97.0}{2}=122 \mathrm{sec}^{-1} \\
& f_{6}=\frac{1024}{2 \pi} \sin \frac{124.5}{2}=145 \mathrm{sec} .^{-1} \\
& f_{7}=\frac{1024}{2 \pi} \sin \frac{152}{2}=158 \mathrm{sec} .^{-1} .
\end{aligned}
$$

\begin{tabular}{|c|c|c|c|c|c|c|c|}
\hline CRANK & $\begin{array}{c}r=1 \\
1 \mathrm{ST} \\
\text { MODE }\end{array}$ & $\begin{array}{c}r=2 \\
2 \mathrm{ND} \\
\text { MONE }\end{array}$ & $\begin{array}{c}r=3 \\
3 \mathrm{RD} \\
\mathrm{MODE}\end{array}$ & $\begin{array}{c}r=4 \\
\text { 4TH } \\
\text { MODE }\end{array}$ & $\begin{array}{c}r=5 \\
5 \mathrm{TH} \\
\text { MODE }\end{array}$ & $\begin{array}{c}r=6 \\
6 T H \\
M O D E\end{array}$ & $\begin{array}{c}r=7 \\
7 \text { TH } \\
\text { MODE }\end{array}$ \\
\hline$x=1$ & 0.999 & 0.990 & 933 & 819 & 662 & 466 & 241 \\
\hline$x=2$ & 996 & 916 & 453 & -258 & -819 & -994 & -682 \\
\hline$x=3$ & 991 & .773 & -258 & -996 & -469 & 656 & 939 \\
\hline$x=4$ & .983 & .573 & -838 & -422 & 933 & 087 & -987 \\
\hline$x=5$ & .972 & .325 & -987 & 707 & 241 & -945 & 798 \\
\hline$x=6$ & .959 & .061 & -629 & 906 & -992 & 819 & -422 \\
\hline
\end{tabular}

In this case we obtain all seven natural frequencies of the system. The modes of vibration in the crankshaft are easily calculated from
Table III.

formula (2.12). Since $K_{1}=0$ we have

$$
\tan \beta=\frac{\sin \mu}{1-\cos \mu}=\cot \frac{\mu}{2} \text {. }
$$

Hence $\beta=\pi / 2-\mu / 2$ and the modes are given by

$$
\theta_{x}=\sin \left(\mu x+\frac{\pi}{2}-\frac{\mu_{r}}{2}\right)=\cos \left[\frac{2 x-1}{2} \mu_{r}\right] .
$$

The values are plotted in Fig. 9 and recorded in Table III.

\section{APPENDIX}

The solution (2.4) is based on the following trigonometrical identities

$$
\begin{aligned}
& \sin (x+1) \mu-2 \sin x \mu \cos \mu+\sin (x-1) \mu=0 \\
& \cos (x+1) \mu-2 \sin x \mu \cos \mu+\cos (x-1) \mu=0 .
\end{aligned}
$$

If we choose $\mu$ so that

$$
\text { or } \begin{aligned}
\cos \mu & =1-\frac{1}{2} \alpha^{2} \\
\alpha & =2 \sin \mu / 2,
\end{aligned}
$$

we may verify from the identities that the expression

satisfies the $n-2$ equations,

$$
\theta_{x}=A \cos \mu x+B \sin \mu x
$$

$$
\theta_{x-1}-\left(2-\alpha^{2}\right) \theta_{x}+\theta_{x+1}=0,
$$

whatever the values of the constants $A$ and $B$. These constants may be adjusted in such a way that expression (c) also satisfies the Eq. (2.3) which may be written

$$
\begin{aligned}
\theta_{2}-2 \theta_{1} \cos \mu-M \theta_{1} & =0 \\
\theta_{n-1}-2 \theta_{n} \cos \mu-N \theta_{n} & =0 .
\end{aligned}
$$

Substitution of expression (c) in these equations yield

$$
\begin{array}{r}
A[\cos 2 \mu-2 \cos \mu \cos \mu-M \cos \mu]+B[\sin 2 \mu-2 \sin \mu \cos \mu-M \sin \mu]=0 \\
A[\cos (n-1) \mu-2 \cos n \mu \cos \mu-N \cos n \mu]+B[\sin (n-1) \mu-2 \sin n \mu \cos \mu-N \sin \mu]=0 .
\end{array}
$$

This may be simplified by taking identities (a) in to account

$$
\begin{aligned}
\cos 2 \mu-2 \cos \mu \cos \mu & =1 \\
\sin 2 \mu-2 \sin \mu \cos \mu & =0 \\
\cos (n-1) \mu-2 \cos n \mu \cos \mu & =\cos (n+1) \mu \\
\sin (n-1) \mu-2 \sin n \mu \cos \mu & =\sin (n+1) \mu .
\end{aligned}
$$

Equations (f) then take the form (2.6) in the text. 